\title{
ASYMPTOTIC BEHAVIOR OF NONLINEAR VOLTERRA DIFFERENCE SYSTEMS
}

\author{
Sung Kyu Choi, Yoon Hoe Goo, and Nam Jip Koo
}

\begin{abstract}
We study the asymptotic behavior of nonlinear Volterra difference system

$$
x(n+1)=f(n, x(n))+\sum_{s=n_{0}}^{n} g(n, s, x(s)), x\left(n_{0}\right)=x_{0}
$$

by using the resolvent matrix $R(n, m)$ of the corresponding linear Volterra system and the comparison principle.
\end{abstract}

\section{Introduction}

To obtain an asymptotic relationship between difference systems Poincaré (1885) and Perron (1921) began to study the long-time behavior of difference systems. The notions of asymptotic equilibrium and asymptotic equivalence become the main objectives in the theory of asymptotic behavior for difference systems. Two difference systems are said to be asymptotically equivalent if, corresponding to each solution of one system, there exists a solution of the other system such that the difference between these two solutions tends to zero. If we know that two systems are asymptotically equivalent, and if we also know the asymptotic behavior of the solutions of one of the systems, then it is clear that we can obtain information about the asymptotic behavior of the solutions of the other systems [2].

For the study of the asymptotic behavior of difference systems, we refer to Choi et al. [5], Medina [9], Medina and Pinto [10], and Trench [11]. Also, Choi et al. [3, 4], Cuevas and Pinto [6], and Zouyousefain and Leela [12] studied the asymptotic behavior of Volterra difference systems.

In this paper we investigate asymptotic equilibrium of nonlinear Volterra difference system. To do this we develop the variation of constants formula for the quasi-linearized Volterra system by Zouyousefain and Leela [12] and

Received September 12, 2006.

2000 Mathematics Subject Classification. 34D05, 34D23, 34E10, 39A11.

Key words and phrases. asymptotic equivalence, asymptotic equilibrium, nonlinear Volterra difference system, resolvent matrix, comparison principle.

This work is supported by the Korea Research Foundation Grant founded by the Korea Government(MOEHRD)(KRF-2005-070-C00015). 
then use the comparison principle which is one of the most efficient methods of obtaining information on the behavior of solutions of difference systems, even when they cannot be solved explicitly.

\section{Main result}

We consider the nonlinear Volterra difference system

$$
x(n+1)=f(n, x(n))+\sum_{s=n_{\mathbf{0}}}^{n} g(n, s, x(s)), x\left(n_{0}\right)=x_{0},
$$

where $f: \mathbb{N}\left(n_{0}\right) \times \mathbb{R}^{m} \rightarrow \mathbb{R}^{m}, g: \mathbb{N}\left(n_{0}\right) \times \mathbb{N}\left(n_{0}\right) \times \mathbb{R}^{m} \rightarrow \mathbb{R}^{m}, \mathbb{N}\left(n_{0}\right)=\left\{n_{0}, n_{0}+\right.$ $\left.1, \ldots, n_{0}+k, \ldots\right\}, n_{0}$ is a nonnegative integer, and $\mathbb{R}^{m}$ is the $m$-dimensional real euclidean space with any convenient norm $|\cdot|$. We assume that $f_{x}=\frac{\partial f}{\partial x}$ and $g_{x}=\frac{\partial g}{\partial x}$ exist and they are continuous and invertible on $\mathbb{N}\left(n_{0}\right) \times \mathbb{R}^{m}$ and $\mathbb{N}\left(n_{0}\right) \times \mathbb{N}\left(n_{0}\right) \times \mathbb{R}^{m}$, respectively. Also, we suppose that $f(n, 0)=0$ and $g(n, s, 0)=0$ for every $n \geq s \geq n_{0}$. Then one associated variational Volterra system of (2.1) is given by

$$
v(n+1)=f_{x}(n, 0) v(n)+\sum_{s=n_{0}}^{n} g_{x}(n, s, 0) v(s)
$$

and a fundamental matrix solution $\Phi\left(n, n_{0}, 0\right)$ of $(2.2)$ is defined by

$$
\Phi\left(n, n_{0}, 0\right)=\frac{\partial x\left(n, n_{0}, 0\right)}{\partial x_{0}}
$$

$[1,7]$. Denote $x(n)=x\left(n, n_{0}, x_{0}\right)$ by the solution of $(2.1)$ with $x\left(n_{0}, n_{0}, x_{0}\right)=$ $x_{0}$.

System (2.1) is said to have asymptotic equilibrium if there exist a single $\xi \in \mathbb{R}^{m}$ and $r>0$ such that any solution $x(n)$ of $(2.1)$ with $x_{0}<r$ satisfies

$$
x(n)=\xi+o(1) \text { as } n \rightarrow \infty
$$

and for every vector in $\mathbb{R}^{m}$, there exists a solution of $(2.1)$ such that the asymptotic relationship (2.3) holds.

Consider the linear Volterra difference system

$$
x(n+1)=A(n) x(n)+\sum_{s=n_{0}}^{n} B(n, s) x(s), x\left(n_{0}\right)=x_{0},
$$

and its perturbation

$$
y(n+1)=A(n) y(n)+\sum_{s=n_{0}}^{n} B(n, s) y(s)+g(n), y\left(n_{0}\right)=x_{0}
$$

where $A(n)$ and $B(n, s)$ are $m \times m$ matrix functions on $\mathbb{N}\left(n_{0}\right)$ and $\mathbb{N}\left(n_{0}\right) \times \mathbb{N}\left(n_{0}\right)$, respectively, and $g(n)$ is a vector function on $\mathbb{N}\left(n_{0}\right)$.

For the nonlinear Volterra difference system (2.1), we set

$$
f_{x}(n, 0)=A(n) \text { and } g_{x}(n, s, 0)=B(n, s) \text { for } n \geq s \geq n_{0} .
$$


Then, by using the mean value theorem, (2.1) can be written as

$$
\begin{aligned}
x(n+1)= & A(n) x(n)+\sum_{s=n_{0}}^{n} B(n, s) x(s)+F(n, x(n)) \\
& +\sum_{s=n_{0}}^{n} G(n, s, x(s)), x\left(n_{0}\right)=x_{0},
\end{aligned}
$$

where

$$
\begin{aligned}
F(n, x) & =\int_{0}^{1}\left[f_{x}(n, x \theta)-f_{x}(n, 0)\right] d \theta \cdot x, \\
G(n, s, x) & =\int_{0}^{1}\left[g_{x}(n, s, x \theta)-g_{x}(n, s, 0)\right] d \theta \cdot x .
\end{aligned}
$$

Let $S\left(\mathbb{N}\left(n_{0}\right), \mathbb{R}^{m}\right)$ be the set of all sequences $y: \mathbb{N}\left(n_{0}\right) \rightarrow \mathbb{R}^{m}$. We assume that the operator $T$ defined on $S\left(\mathbb{N}\left(n_{0}\right), \mathbb{R}^{m}\right)$ satisfies the following :

(i) $|T|: S\left(\mathbb{N}\left(n_{0}\right), \mathbb{R}^{+}\right) \rightarrow S\left(\mathbb{N}\left(n_{0}\right), \mathbb{R}^{+}\right)$is nondecreasing.

(ii) For any $y(n)$ and $z(n)$ in $S\left(\mathbb{N}\left(n_{0}\right), \mathbb{R}^{m}\right),|y(n)| \leq|z(n)|$ for each $n_{0} \leq$ $n \leq n_{1}$ implies $|T y(n)| \leq|T z(n)|$,

(iii) $|T y(n)| \leq|T||y(n)|$ for any $y(n) \in S\left(\mathbb{N}\left(n_{0}\right), \mathbb{R}^{m}\right)$. If we impose on $T$ various meanings, there will be appear various types of equations [9].

The following is the most basic comparison principle.

Lemma 2.1. [8, Theorem 1.6.1] Let $g(n, r)$ be a function nondecreasing with respect $r \geq 0$ for any fixed $n \in \mathbb{N}\left(n_{0}\right)$. Suppose that for $n \geq n_{0}$, the inequalities

$$
\begin{aligned}
& v(n+1) \leq g(n, v(n)), \\
& u(n+1) \geq g(n, u(n))
\end{aligned}
$$

hold. Then $v\left(n_{0}\right) \leq u\left(n_{0}\right)$ implies $v(n) \leq u(n)$ for all $n \geq n_{0}$.

We need the following comparison principle which is a modification of Lemma 2.1 .

Lemma 2.2. Let $\iota(n, r, u)$ be a nondecreasing function in $r$ and $u$ for any fixed $n \in \mathbb{N}\left(n_{0}\right)$. Suppose that for $n \geq n_{0}$,

$$
v(n)-\sum_{l=n_{0}}^{n-1} \iota(l, v(l),|T| v(l))<u(n)-\sum_{l=n_{0}}^{n-1} \iota(l, u(l),|T| u(l)) .
$$

If $v\left(n_{0}\right)<u\left(n_{0}\right)$, then $v(n)<u(n)$ for all $n \geq n_{0}$.

Proof. If we assume that this is not the case, then we have $v(k)=u(k)$ and $v(l)<u(l)$ for some $k \in \mathbb{N}\left(n_{0}\right)$ with $n_{0} \leq l<k$. Since $|T| v(l) \leq|T| u(l)$ for $n_{0} \leq l<k$, we obtain

$$
v(k)<u(k)+\sum_{l=n_{0}}^{k-1} \iota(l, v(l),|T| v(l))-\sum_{l=n_{0}}^{k-1} \iota(l, u(l),|T| u(l)) \leq u(k),
$$

which is a contradiction. 
The following lemma states a result corresponding to Fubini's theorem, which can be proved by the induction.

Lemma 2.3. [12] Let $L(n, s)$ and $K(n, s)$ be $m \times m$ matrices defined for $s, n \geq n_{0}$ such that $L$ and $K$ are zero matrices for $s, n \leq n_{0}$. Then the relation

$$
\sum_{s=n_{0}}^{n-1} L(n, s+1) \sum_{\sigma=n_{0}}^{s-1} K(s, \sigma) x(\sigma)=\sum_{s=n_{0}}^{n-1} \sum_{\sigma=s+1}^{n-1} L(n, \sigma+1) K(\sigma, s) x(s)
$$

holds, where $x: \mathbb{N}\left(n_{0}\right) \rightarrow \mathbb{R}^{m}$ is a vector function.

We define the resolvent matrix $R(n, m)$ of the linear system (2.4) as the unique solution of the matrix difference equation

$$
R(n, m)=R(n, m+1) A(m)+\sum_{r=m}^{n-1} R(n, r+1) B(r, m), n-1 \geq m \geq n_{0}
$$

with $R(m, m)=I$ [7]. Then we can obtain the expression of a solution of the perturbation (2.5) via the following variation of constants formula.

Theorem 2.4. The unique solution $y\left(n, n_{0}, y_{0}\right)$ of $(2.5)$ satisfying $y\left(n_{0}\right)=y_{0}$ is given by

$$
y\left(n, n_{0}, y_{0}\right)=R\left(n, n_{0}\right) y_{0}+\sum_{s=n_{0}}^{n-1} R(n, s+1) g(s),
$$

where $R(n, m)$ is the unique solution of the matrix difference equation (2.7).

Proof. Letting $p(s)=R(n, s) y(s), n-1 \geq s \geq n_{0}$, we have

$$
\begin{aligned}
\Delta p(s)= & R(n, s+1) y(s+1)-R(n, s) y(s) \\
= & {[R(n, s+1) A(s)-R(n, s)] y(s) } \\
& +R(n, s+1) \sum_{r=n_{0}}^{s} B(s, r) y(r)+R(n, s+1) g(s) .
\end{aligned}
$$

Summing both sides of (2.9) from $n-1$ to $n_{0}$, we obtain, by Lemma 2.3 ,

$$
\begin{aligned}
p(n)= & y(n)=R\left(n, n_{0}\right) y_{0}+\sum_{s=n_{0}}^{n-1} R(n, s+1) g(s) \\
& +\sum_{s=n_{0}}^{n-1}[R(n, s+1) A(s)-R(n, s)] y(s) \\
& +\sum_{s=n_{0}}^{n-1} R(n, s+1) \sum_{r=n_{0}}^{s-1} B(s, r) y(r)+\sum_{s=n_{0}}^{n-1} R(n, s+1) B(s, s) y(s)
\end{aligned}
$$




$$
\begin{aligned}
= & R\left(n, n_{0}\right) y_{0}+\sum_{s=n_{0}}^{n-1} R(n, s+1) g(s) \\
& +\sum_{s=n_{0}}^{n-1}\left[R(n, s+1) A(s)-R(n, s)+\sum_{r=s}^{n-1} R(n, r+1) B(r, s)\right] y(s) .
\end{aligned}
$$

From (2.7) we have the result

$$
y(n)=R\left(n, n_{0}\right) y_{0}+\sum_{s=n_{0}}^{n-1} R(n, s+1) g(s) .
$$

Example 2.5. [4] We consider the linear Volterra difference equation

$$
\begin{aligned}
x(n+1) & =A(n) x(n)+\sum_{s=n_{0}}^{n} B(n, s) x(s) \\
& =2 x(n)+\sum_{s=n_{0}}^{n} 2^{n-s} x(s),
\end{aligned}
$$

where $A(n)=2$ and $B(n, s)=2^{n-s}$. Then any solution $x\left(n, n_{0}, x_{0}\right)$ of $(2.10)$ through the initial point $x\left(n_{0}, n_{0}, x_{0}\right)=x_{0}$ is given by

$$
x\left(n, n_{0}, x_{0}\right)=\frac{x_{0}}{3}\left[1+2 \cdot 4^{n-n_{0}}\right], n \in \mathbb{N}\left(n_{0}\right) .
$$

Thus the resolvent matrix solution $R(n, m)$ with $R(m, m)=1$ of the difference equation

$$
\begin{aligned}
R(n, m) & =R(n, m+1) A(n)+\sum_{r=m}^{n-1} R(n, r+1) B(r, m) \\
& =R(n, m+1) 2+\sum_{r=m}^{n-1} R(n, r+1) 2^{r-m}, n-1 \geq m \geq n_{0},
\end{aligned}
$$

is given by

$$
R(n, m)=\frac{1}{3}\left[1+2 \cdot 4^{n-m}\right] .
$$

It follows from the simple calculation that

$$
\begin{aligned}
& R(n, m+1) A(n)+\sum_{r=m}^{n-1} R(n, r+1) B(r, m) \\
= & \frac{2}{3}\left[1+2 \cdot 4^{n-m-1}\right]+\sum_{r=m}^{n-1} \frac{1}{3}\left[1+2 \cdot 4^{n-r-1}\right] 2^{r-m} \\
= & \frac{1}{3}\left[2+4^{n-m}\right]+\frac{1}{3}\left[2^{n-m}-1\right]+\frac{2^{2 n-m-1}}{3}\left[2^{1-m}-2^{1-n}\right]
\end{aligned}
$$




$$
\begin{aligned}
& =\frac{1}{3}\left[1+2 \cdot 4^{n-m}\right] \\
& =R(n, m) .
\end{aligned}
$$

In fact, we note that the fundamental matrix of (2.10) is given by

$$
\frac{\partial x\left(n, n_{0}, x_{0}\right)}{\partial x_{0}}=\Phi\left(n, n_{0}\right)=\frac{1}{3}\left[1+2 \cdot 4^{n-n_{0}}\right]
$$

and

$$
\Phi\left(n, n_{0}\right)=R\left(n, n_{0}\right) .
$$

Remark 2.6. In the special case when $f(n, x)=A(n) x(n)$ and $g(n, s, x)=$ $B(n, s) x(s)$ in the nonlinear system (2.1) we note that the resolvent matrix $R(n, m)$ for Equation (2.7) is closely related to the fundamental matrix $\Phi\left(n, n_{0}\right)$. By the uniqueness of solution, it is easy to see that $R\left(n, n_{0}\right)=\Phi\left(n, n_{0}\right)$, and $R(n, m)=\Phi(n-m)$ for the equation of convolution type such as $B(n, s)=$ $B(n-s)$.

The following main result can be obtained by improving the scalar Volterra difference equation in Theorem 3.2 in [4].

Theorem 2.7. Assume that

(i) (2.4) has asymptotic equilibrium,

(ii) $F$ and $G$ perturbed terms in (2.6) satisfies

$$
\begin{aligned}
& |F(n, x)| \leq \omega_{1}(n,|x|),|G(n, s, x)| \leq \omega_{2}(n, s,|x|), n \geq s \geq n_{0}, x \in \mathbb{R}^{m}, \\
& \text { where } \omega_{1}: \mathbb{N}\left(n_{0}\right) \times \mathbb{R}^{+} \rightarrow \mathbb{R}^{+}, \omega_{2}: \mathbb{N}\left(n_{0}\right) \times \mathbb{N}\left(n_{0}\right) \times \mathbb{R}^{+} \rightarrow \mathbb{R}^{+} \text {, and } \\
& \omega_{1}(n, u) \text { and } \omega_{2}(n, s, u) \text { are continuous and nondecreasing in } u \text { for } n \\
& \text { and }(n, s) \text {, respectively. } \\
& \text { Also, we consider the scalar Volterra difference equation } \\
& u(n+1)=u(n)+M\left[\omega_{1}(n, u(n))\right. \\
& \left.+\sum_{s=n_{0}}^{n} \omega_{2}(n, s, u(s))\right], u\left(n_{0}\right)=u_{0}>0,
\end{aligned}
$$

where $M$ is an upper bounded number of $R(n, m)$.

(iii) All solutions of (2.11) are bounded on $\mathbb{N}\left(n_{0}\right)$.

Then (2.1) has asymptotic equilibrium provided $M\left|x_{0}\right|<u_{0}$.

Proof. Let $x(n)$ be any solution of (2.1). From the variation of constants formula, and (i)-(ii), we obtain

$$
\begin{aligned}
|x(n)| & =\left|R\left(n, n_{0}\right) x_{0}+\sum_{s=n_{0}}^{n-1} R(n, s+1)\left[F(s, x(s))+\sum_{\sigma=n_{0}}^{s} G(s, \sigma, x(\sigma))\right]\right| \\
& \leq M\left|x_{0}\right|+M \sum_{s=n_{0}}^{n-1}\left[\omega_{1}(s,|x(s)|)+\sum_{\sigma=n_{0}}^{s} \omega_{2}(s, \sigma,|x(\sigma)|)\right],
\end{aligned}
$$


where $M$ is a constant from the boundedness of $R(n, m)$ for each $n \geq m \geq t_{0}$ and $d=M\left|x_{0}\right|$, we have the following difference inequality :

$$
\begin{aligned}
& |x(n)|-M \sum_{s=n_{0}}^{n-1}\left[\omega_{1}(s,|x(s)|)+\sum_{\sigma=n_{0}}^{s} \omega_{2}(s, \sigma,|x(\sigma)|)\right] \\
\leq & d \\
< & u_{0} \\
= & u(n)-M \sum_{s=n_{0}}^{n-1}\left[\omega_{1}(s, u(s))+\sum_{\sigma=n_{0}}^{s} \omega_{2}(s, \sigma, u(\sigma))\right] .
\end{aligned}
$$

Setting $\iota(n, s, u)=M\left[\omega_{1}(n, u)+|T| u\right]$ with $|T| u(n)=\sum_{s=n_{0}}^{n} \omega_{2}(n, s, u(s))$, it follows from Lemma 2.2 that

$$
|x(n)|<u(n), \text { for each } n \geq n_{0}
$$

provided $d<u_{0}$.

Now, we prove that the solution $x(n)$ of $(2.1)$ converges to a vector as $n \rightarrow \infty$. Consider the sequence

$$
v\left(n, n_{0}, x_{0}\right)=\sum_{s=n_{0}}^{n-1} R(n, s+1)\left[F(s, x(s))+\sum_{\sigma=n_{0}}^{s} G(s, \sigma, x(\sigma))\right] .
$$

By using the monotonicity of $\omega_{1}, \omega_{2}$, and asymptotic equilibrium of (2.4), we have

$$
\begin{aligned}
\left|v(n)-v\left(n_{1}\right)\right| & \leq\left|\sum_{s=n_{1}}^{n-1} R(n, s+1)\left[F(s, x(s))+\sum_{\sigma=n_{0}}^{s} G(s, \sigma, x(\sigma))\right]\right| \\
& \leq M \sum_{s=n_{1}}^{n-1}\left[\omega_{1}(s,|x(s)|)+\sum_{\sigma=n_{0}}^{s} \omega_{2}(s, \sigma,|x(\sigma)|)\right] \\
& \leq M \sum_{s=n_{1}}^{n-1}\left[\omega_{1}(s, u(s))+\sum_{\sigma=n_{0}}^{s} \omega_{2}(s, \sigma, u(\sigma))\right] \\
& \leq u(n)-u\left(n_{1}\right),
\end{aligned}
$$

for any $n \geq n_{1} \geq n_{0}$. Since $u(n)$ has the Cauchy property, $v(n)$ converges to a vector $v_{\infty}\left(n_{0}, x_{0}\right)$ as $n \rightarrow \infty$. Hence the solution $x(n)$ of $(2.1)$ tends to a finite limit vector $\xi$ as $n \rightarrow \infty$, i.e., $\xi=R_{\infty} x_{0}+v_{\infty}\left(n_{0}, x_{0}\right)$.

By the same method of Medina in [9] we can be prove that the converse asymptotic relationship holds.

Acknowledgement. The authors are grateful to the referees for their useful comments. 


\section{References}

[1] R. P. Agarwal, Difference Equations and Inequalities, Theory, methods, and applications. Second edition. Monographs and Textbooks in Pure and Applied Mathematics, 228. Marcel Dekker, Inc., New York, 2000.

[2] F. Brauer, Asymptotic equivalence and asymptotic behaviour of linear systems, Michigan Math. J. 9 (1962), 33-43.

[3] S. K. Choi and N. J. Koo, Asymptotic equivalence between two linear Volterra difference systems, Comput. Math. Appl. 47 (2004), no. 2-3, 461-471.

[4] S. K. Choi, N. J. Koo, and Y. H. Goo, Asymptotic property of nonlinear Volterra difference systems, Nonlinear Anal. 51 (2002), no. 2, Ser. A: Theory Methods, 321-337.

[5] S. K. Choi, N. J. Koo, and H. S. Ryu, Asymptotic equivalence between two difference systems, Advances in difference equations, IV., Comput. Math. Appl. 45 (2003), no. 6-9, $1327-1337$.

[6] C. Cuevas and M. Pinto, Asymptotic behavior in Volterra difference systems with unbounded delay, Fixed point theory with applications in nonlinear analysis, J. Comput. Appl. Math. 113 (2000), no. 1-2, 217-225.

[7] S. Elaydi, Periodicity and stability of linear Volterra difference systems, J. Math. Anal. Appl. 181 (1994), no. 2, 483-492.

[8] V. Lakshmikantham and D. Trigiante, Theory of difference equations, Numerical methods and applications. Mathematics in Science and Engineering, 181. Academic Press, Inc., Boston, MA, 1988.

[9] M. Medina, Asymptotic properties of solutions of nonlinear difference equations, J. Comput. Appl. Math. 70 (1996), no. 1, 57-66.

[10] R. Medina and M. Pinto, Asymptotic equivalence and asymptotic behavior of difference systems, Commun. Appl. Anal. 1 (1997), no. 4, 511-523.

[11] W. F. Trench, Linear asymptotic equilibrium and uniform, exponential, and strict stability of linear difference systems, Advances in difference equations, II. Comput. Math. Appl. 36 (1998), no. 10-12, 261-267.

[12] M. Zouyousefain and S. Leela, Stability results for difference equations of Volterra type, Appl. Math. Comput. 36 (1990), no. 1, part I, 51-61.

\section{Sung KYu Chor}

DEPARTMENT OF MATHEMATICS

Chungnam National University

DAEJEON 305-764, KoREA

E-mail address: skchoi@math.cnu.ac.kr

YOON HOE GOO

Department OF MATHEMATiCs

HANSEO UNIVERSITY

SEOSAN 352-820, KoREA

E-mail address: yhgoo@hanseo.ac.kr

NAM JIP KOO

Department of Mathematics

Chungnam National University

DAEJEON 305-764, KorEA

E-mail address: njkoo@math. cnu.ac.kr 